\title{
A hybrid image retrieval system for microscopy images
}

\author{
Weixin Jiang ${ }^{1}$, Eric Schwenker ${ }^{2}$, Trevor Spreadbury ${ }^{2}$, Oliver Cossairt ${ }^{1}$ and Maria KY Chan ${ }^{3}$ \\ ${ }^{1}$ Northwestern University, United States, ${ }^{2}$ Argonne National Laboratory, United States, ${ }^{3}$ ANL, United \\ States
}

Scientific literature provides the authoritative data source for most scientific fields. Numerous microscopy images rich in information, in particular, are contained within scientific publications. The retrieval of these imaging data currently requires a keyword search and labor-intensive human reading of individual articles. An efficient image retrieval tool would be significantly beneficial for researchers.

Information retrieval [5] engines have been widely used for searching scientific articles with one or a few keywords. However, such tools are not accurate and efficient for image retrieval because images in scientific literature are not always well labeled by textual description. To this end, we propose a hybrid image retrieval tool to retrieve images from scientific literature. In the proposed system, we take advantage of recent progress in content-based image retrieval approaches $[4,8,9,10]$, which directly measure the similarity between images by encoding images into fix-length feature vectors. Specifically, the proposed image retrieval system uses both the content of images and textual information (extracted from the image or given from the user) for image retrieval. First, keywords provided by the user are fed into the Image Acquisition Module, which selects relevant articles from different journals and extracts candidate images to build the database. Then both the query and all the images in the collected database are fed into the Image Analysis Module, which encodes the content of images into feature vector and extracts important textual information from the images. Finally, we compute the distance between the query image and the images in the database with both visual information and texture information and retrieve the most similar ones.

More details about the proposed image retrieval system are shown in Fig. 1. With the keyword provided by the user (e.g. "nano"), we apply the EXSCLAIM! [1,2] Pipeline to search relevant scientific articles from journals (e.g. Nature, ACS family) and extract microscopy images (e.g. $\sim 64 \mathrm{k}$ ) as candidate images to build the database. Then we build the image analysis module with an off-the-shelf feature extractor [3, 4] and a scale bar detector [6, 7]. The feature extractor encodes the content of the image into a 2048-d feature vector and the scale bar detector detects the scale bar information from each image. As shown in Fig. 2, we first sort the candidates with the visual distance (e.g. cosine distance between feature vectors) between images and then use the scale bar information to re-rank the candidates.

As future work, caption information will be included in the hybrid image retrieval system, since authors tend to include essential description in the caption. For materials imaging data, retrieving images with only the content of the image is not sufficient to give confident results. Different materials may look visually similar in a certain scale. In this paper, we use scale bar information to eliminate the candidates that are measured in a different scale, but it could fail in case when scale bar information is not available 
in the image. Thus, incorporating the information from the captions could provide extra information to improve the performance of the image retrieval system.

\section{Acknowledgements:}

This material is based upon work supported by Laboratory Directed Research and Development (LDRD) funding from Argonne National Laboratory, provided by the Director, Office of Science, of the U.S. Department of Energy under Contract No. DE-AC02-06CH11357. Use of the Center for Nanoscale Materials, an Office of Science user facility, was supported by the U.S. Department of Energy, Office of Science, Office of Basic Energy Sciences, under Contract No. DE-AC02-06CH11357. We gratefully acknowledge the computing resources provided and operated by the Joint Laboratory for System Evaluation (JLSE) at Argonne National Laboratory.

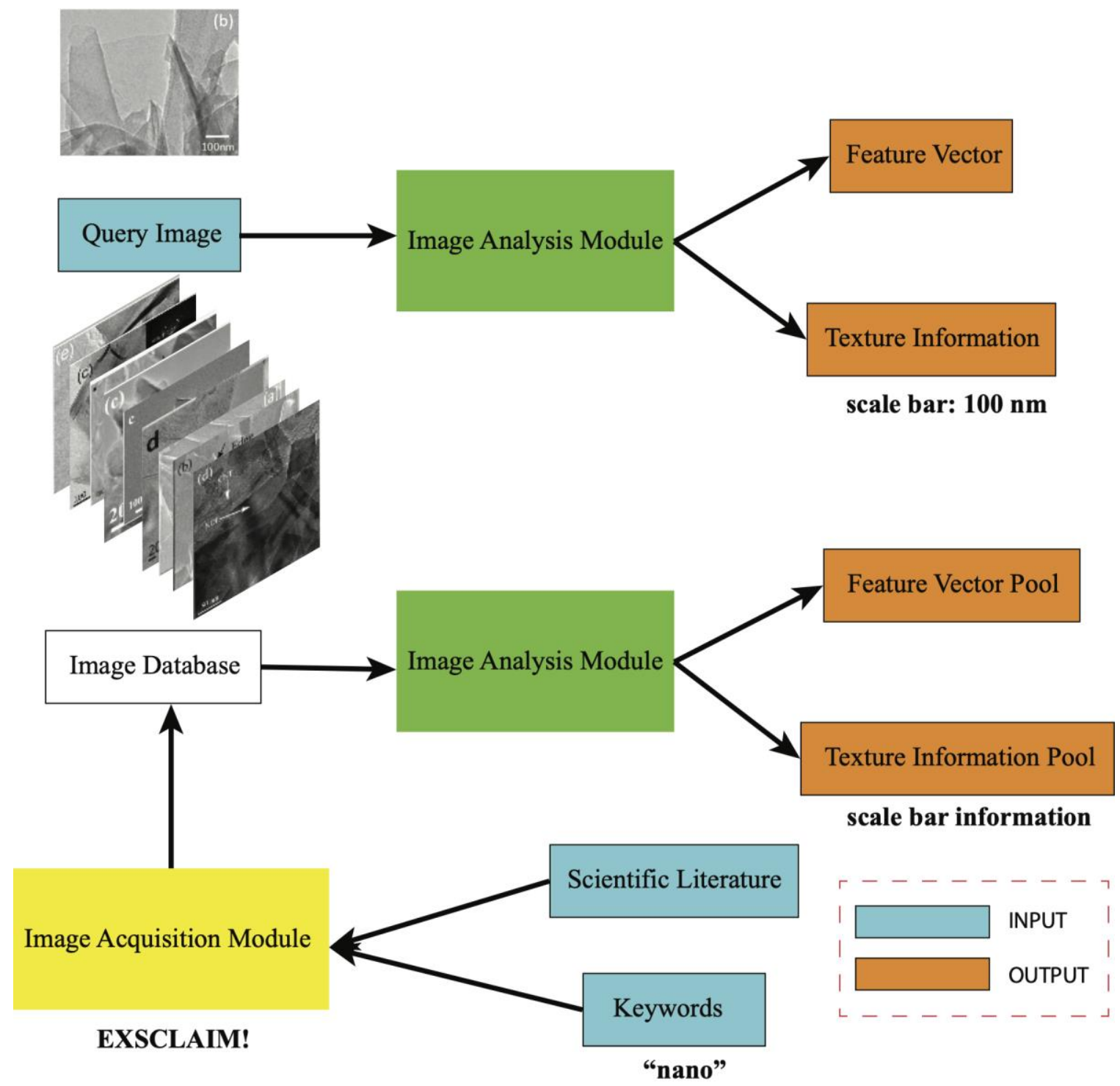

Figure 1. The pipeline of the proposed image retrieval system. 


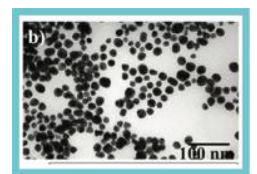

Query Image

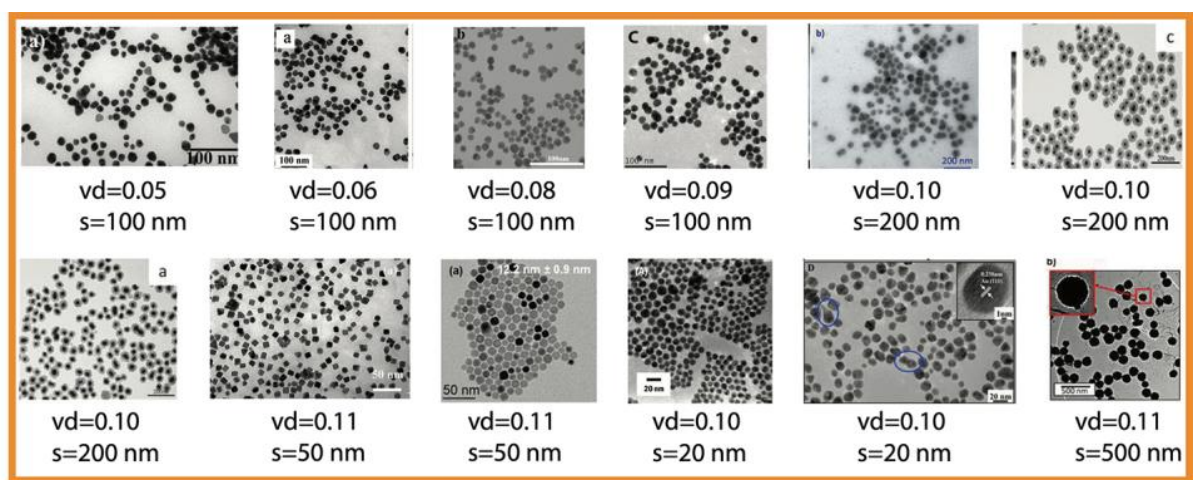

Retrieved Images

Figure 2. An example of the image retrieval system. "vd" and "s" denotes the visual distance and scale bar label, respectively.

\section{References}

[1] Schwenker, E., Jiang, W., Spreadbury, T., O'Brien, S., Ferrier, N., Cossairt, O., \& Chan, M. (2020). Constructing Self-Labeled Materials Imaging Datasets from Open Access Scientific Journals with EXSCLAIM! Microscopy and Microanalysis, 26(S2), 3096-3097. doi:10.1017/S1431927620023806 [2] Jiang, W., Schwenker, E., Spreadbury, T., Ferrier, N., Chan, M. K., \& Cossairt, O. (2021). A Twostage Framework for Compound Figure Separation. arXiv preprint arXiv:2101.09903.

[3] Radenović, F., Tolias, G., \& Chum, O. (2018). Fine-tuning CNN image retrieval with no human annotation. IEEE transactions on pattern analysis and machine intelligence, 41(7), 1655-1668.

[4] Radenović, F., Tolias, G., \& Chum, O. (2016, October). CNN image retrieval learns from BoW: Unsupervised fine-tuning with hard examples. In European conference on computer vision (pp. 3-20). Springer, Cham.

[5] Sanderson, M., \& Croft, W. B. (2012). The history of information retrieval research. Proceedings of the IEEE, 100(Special Centennial Issue), 1444-1451.

[6] Ren, S., He, K., Girshick, R., \& Sun, J. (2015). Faster r-cnn: Towards real-time object detection with region proposal networks. arXiv preprint arXiv:1506.01497.

[7] He, K., Zhang, X., Ren, S., \& Sun, J. (2016). Deep residual learning for image recognition. In Proceedings of the IEEE conference on computer vision and pattern recognition (pp. 770-778)

[8] Zheng, L., Yang, Y., \& Tian, Q. (2017). SIFT meets CNN: A decade survey of instance retrieval. IEEE transactions on pattern analysis and machine intelligence, 40(5), 1224-1244.

[9] Hu, J., Lu, J., \& Tan, Y. P. (2014). Discriminative deep metric learning for face verification in the wild. In Proceedings of the IEEE conference on computer vision and pattern recognition (pp. 1875-1882). [10] Hoffer, E., \& Ailon, N. (2015, October). Deep metric learning using triplet network. In International workshop on similarity-based pattern recognition (pp. 84-92). Springer, Cham. 